\title{
Ultrafast optical switching of the THz transmission through metallic subwavelength hole arrays
}

\author{
E. Hendry, ${ }^{1, *}$ M. J. Lockyear, ${ }^{1}$ J. Gómez Rivas, ${ }^{2}$ L. Kuipers, ${ }^{2}$ and M. Bonn ${ }^{2}$ \\ ${ }^{1}$ School of Physics, University of Exeter, Stocker Road, Exeter EX4 4QL, United Kingdom \\ ${ }^{2}$ FOM Institute for Atomic and Molecular Physics, Kruislaan 407, 1098 SJ Amsterdam, The Netherlands
}

(Received 1 February 2007; revised manuscript received 9 April 2007; published 5 June 2007)

\begin{abstract}
We demonstrate ultrafast optical switching of the transmission of terahertz radiation through a metal grating with subwavelength holes. By fabricating the grating on a semiconductor silicon substrate, we are able to control the grating transmission intensity by varying the photodoping level of the silicon and thereby the resonant coupling to the metal grating. As such, we are able to switch the transmission on picosecond time scales with low visible light intensities, observing a factor of 2-5 improvement in photomodulation efficiency at resonance wavelengths over a bare silicon surface.
\end{abstract}

DOI: 10.1103/PhysRevB.75.235305

PACS number(s): 73.20.Mf, 41.20.Jb, 42.25.Fx, 78.47.+p

\section{INTRODUCTION}

Thin metal films with a two-dimensional periodic array of subwavelength holes can exhibit anomalously high transmission at certain wavelengths above the cutoff for the apertures. ${ }^{1}$ This effect has been described in terms of surface waves, such as surface-plasmon polaritons, generated through scattering from the periodic grating, which can propagate along the surface between apertures. The excitation of surface waves lead to a field enhancement at the metal-dielectric interface and a large transmission through subwavelength apertures at distinct wavelengths associated with the geometrical distance in-between apertures. The highly frequency-dependent transmission, as well as the localized electric-field strengths at the metal interface, means that hole arrays are finding applications in visible spectroscopy, ${ }^{2}$ subwavelength optics ${ }^{3}$ nonlinear optics, ${ }^{4}$ and photolithography. ${ }^{5}$ The recent observation of enhanced transmission of $\mathrm{THz}$ (Refs. 6-9) and microwave ${ }^{10,11}$ radiation through metallic arrays of subwavelength apertures has renewed the debate about the physical origin of this phenomenon. Surface-plasmon polaritons are not supported by flat metallic surfaces at low frequencies due to the negligible skin depth of metals at these frequencies. However, it has been proposed that the penetration of the electromagnetic field into the holes leads to a larger effective skin depth and the excitation of spoof surface-plasmon polaritons. ${ }^{12}$ Alternative explanations for the extraordinary transmission in terms of dynamical diffraction theory (involving diffracted surface waves) have also been proposed. ${ }^{13-16}$

At terahertz $(\mathrm{THz})$ frequencies, there is more scope for switching transmission, which is of particular significance for high bandwidth and time-resolved applications. Enhanced transmission through semiconductor ${ }^{17-19}$ hole arrays has recently been reported in the $\mathrm{THz}$ frequency regime. Rivas et $a l^{20}$ have recently demonstrated thermal switching of $\mathrm{THz}$ transmission by modifying with temperature the density of free carriers in a silicon array, while Pan et al. ${ }^{21}$ demonstrated magnetic switching by using a nematic liquid crystal in a metal array. Switching has also been demonstrated by utilizing the metal-insulator transition in vanadium oxide hole arrays. ${ }^{22}$ Optical control of $\mathrm{THz}$ transmission has been demonstrated for a semiconductor hole array, ${ }^{23}$ where photo- excitation using a high repetition rate pulsed laser source resulted in a steady buildup of surface charge densities and presumably a modification of surface modes. All of these control techniques are, however, inapplicable when fast switching is required.

In this paper, we present measurements and modeling of $\mathrm{THz}$ transmission through periodic arrays of subwavelength holes in thin gold films on silicon. Sharp resonances are observed in the transmission spectra at wavelengths related to the array dimensions. By fabricating the metal array on a semiconductor substrate, we are able to finely control the resonant transmission intensities of the grating through above band-gap photoexcitation of the semiconductor substrate. It is shown that the transmission can be switched on picosecond time scales with low intensities of visible light.

\section{EXPERIMENTAL PROCEDURE}

The samples are fabricated by contact lithography on a 1-mm-thick wafer of silicon, followed by the evaporation of a film of $200 \mathrm{~nm}$ of gold and a lift-off process to generate the array of holes. Figure 1(a) shows an optical microscope image of our sample: it consists of a gold layer with square holes (length $a=38 \mu \mathrm{m}$ with lattice spacing $L=100 \mu \mathrm{m}$ ). While a homogeneous gold layer of $200 \mathrm{~nm}$ is completely opaque to $\mathrm{THz}$ radiation, the silicon wafer substrate exhibits around $50 \%$ transmission.

We measure the zero-order transmission of the grating using a time domain $\mathrm{THz}$ spectrometer. ${ }^{24}$ The incident $\mathrm{THz}$ pulses are essentially single cycle electromagnetic pulses of about 1 ps duration and peak field strength of $\sim 1 \mathrm{kV} / \mathrm{cm}$ when focused for detection. The time-dependent field strengths at normal incidence are detected directly in the far field, with and without the sample $\left[E(t)\right.$ and $E_{0}(t)$, respectively-see inset of Fig. 1(b), measured using a collimated beam with a beam diameter $\sim 8 \mathrm{~mm}]$. The spectrometer is flushed with dry nitrogen gas to prevent absorption by water vapor. By Fourier transforming the time wave forms, the intensity transmission spectrum $I(\omega)=|E(\omega)|^{2} /\left|E_{0}(\omega)\right|^{2}$ is calculated-plotted in Fig. 1(b) as a function of wavelength $\lambda$. The spectral resolution of Fig. 1(b) is $66 \mathrm{GHz}$ (or $18 \mu \mathrm{m}$ at $300 \mu \mathrm{m}$ ), corresponding to a time window of $15 \mathrm{ps}$, limited by reflections within the silicon wafer. We observe reso- 
(a)

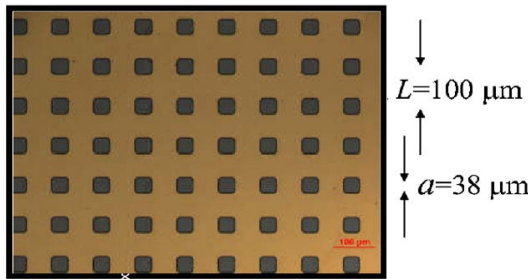

(b)

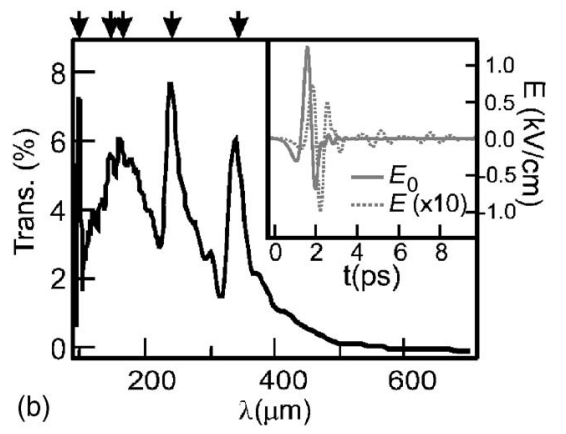

FIG. 1. (Color online) (a) Optical microscope image of the sample, showing gold and silicon (black) regions. (b) The transmission spectrum for plane-wave transmission. The arrows indicate peak wavelengths predicted by Eq. (1). Inset: The time domain measurements of the transmitted and reference electric fields.

nances in Fig. 1(b) at $\lambda \sim 100 \mu \mathrm{m}$ (at the very edge of the measured spectral region) and 150, 170, 250, and $350 \mu \mathrm{m}$.

\section{RESULTS AND DISCUSSION}

The wavelengths of resonant surface waves on a grating interface, excited under normal incidence, are approximately given by ${ }^{3}$

$$
\lambda=\frac{L}{\sqrt{i^{2}+j^{2}}} \sqrt{\frac{\varepsilon_{d} \varepsilon_{m}}{\varepsilon_{d}+\varepsilon_{m}}},
$$

dependent on $i$ and $j$, the integer mode indices, and on the dielectric functions of the dielectric $\left(\varepsilon_{d}\right)$ and metal $\left(\varepsilon_{m}\right)$ at the interfaces. For our gratings, Eq. (1) has two sets of solutions: for the air-gold interface $\left(\varepsilon_{d}=\varepsilon_{\text {air }}, \varepsilon_{m}=\varepsilon_{\text {gold }}\right)$ and for the gold-silicon interface $\left(\varepsilon_{m}=\varepsilon_{\text {gold }}, \varepsilon_{d}=\varepsilon_{\mathrm{Si}}\right)$. At $\mathrm{THz}$ frequencies, $\left|\varepsilon_{\text {gold }}\right| \gg \varepsilon_{\text {air }}=1$ and $\varepsilon_{\mathrm{Si}}=11.9$. The arrows in Fig. 1 (b) (in order of increasing wavelength) represent the $(i=1$, $j=0)$ mode for the air-gold interface, and the $(2,1),(2,0)$, $(1,1)$, and $(1,0)$ modes for the gold-silicon interface. All of the transmission resonances in Fig. 1(b) are at wavelengths significantly larger than the cutoff wavelength $[\sim 76 \mu \mathrm{m}$ for square holes with $a=38 \mu \mathrm{m}$ (Ref. 12)], so the transmission of a single hole should be very small. Despite this, the $(1,1)$ and $(1,0)$ modes for the silicon interface, in particular, give rise to large peaks in our transmission spectrum at 250 and $350 \mu \mathrm{m}$, respectively, similar to previous reports of $\mathrm{THz}$ transmission through metal hole arrays. ${ }^{6-8}$

We can optically modify the $\mathrm{THz}$ dielectric function of the silicon with above band-gap photoexcitation [indirect gap of $\sim 1.1 \mathrm{eV}$, direct gap of $\sim 3.2 \mathrm{eV}$ (Ref. 25)] of free charge carriers. This photoexcitation is achieved using either 1.5 or $3.1 \mathrm{eV}$ photons in single shot, 150 fs laser pulses, which permits switching on ultrafast time scales. To allow spatial overlap of the $\mathrm{THz}$ light with the excitation pulses, we weakly focus the THz pulses onto the sample using parabolic mirrors (focal length of $6 \mathrm{~cm}$, numerical aperture of 0.18 ) resulting in a beam waist of $\sim 1.8 \mathrm{~mm}$ in diameter, allowing the simultaneous illumination of $\sim 300$ holes. Note that, in this configuration, the transmission peaks are somewhat broadened as a result of the distribution of incident wave vectors, as will be detailed in a later contribution.

Figures 2(a) and 2(b) show transmission spectra $10 \mathrm{ps}$
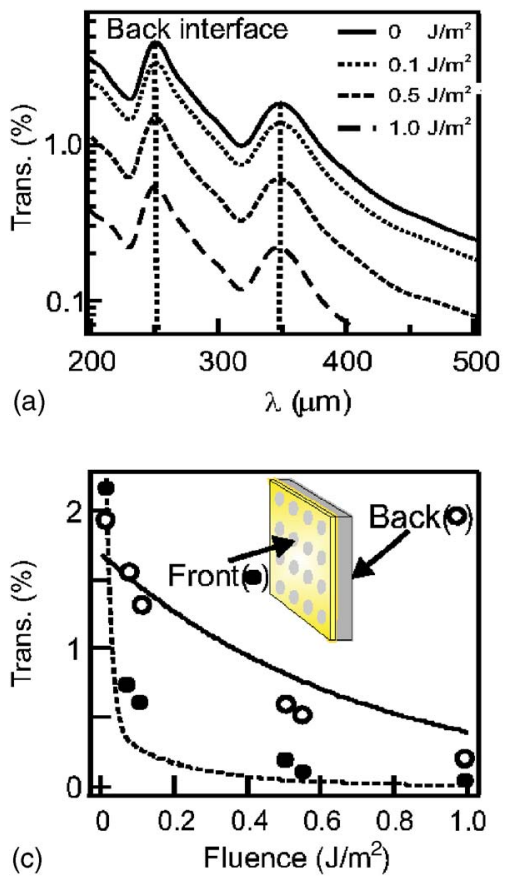
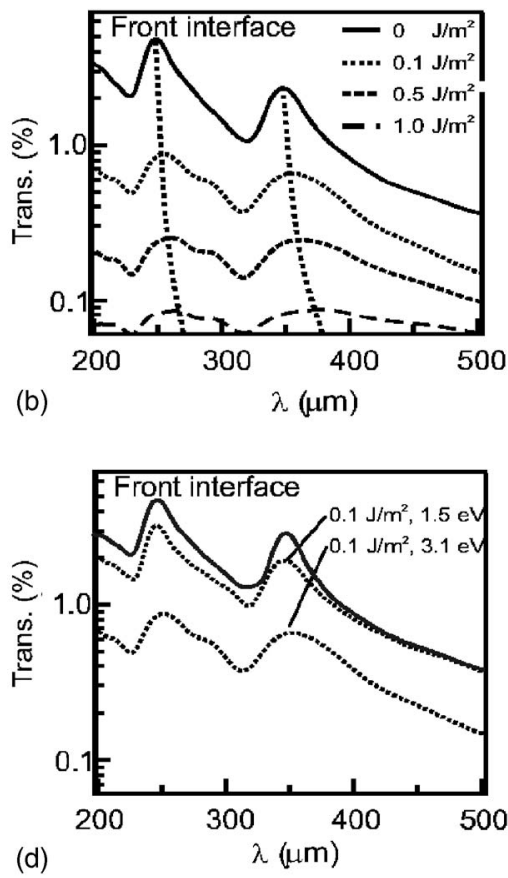

FIG. 2. (Color online) (a) Transmission spectra measured $10 \mathrm{ps}$ after photoexcitation of the back interface of the grating using $3.1 \mathrm{eV}$ photon pulses. (b) Transmission after excitation of the front interface. (c) Dots indicate the absolute transmission at the $(1,0)$ peak at $\lambda=346 \mu \mathrm{m}$ after photoexciting the front (filled) and back (nonfilled) interfaces. The dotted and solid lines are for photoexcitation of the hole array and bare silicon surfaces, respectively. (d) Comparison between excitation of the front interface with $1.5 \mathrm{eV}$ photons and $3.1 \mathrm{eV}$ photons. 
after excitation with $3.1 \mathrm{eV}$ photons of the back and front interfaces of the sample, respectively [as defined in the inset of Fig. 2(c)]. The penetration depth of $3.1 \mathrm{eV}$ light [ $\sim 125 \mathrm{~nm}$ (Ref. 25$)]$ is significantly smaller than the thickness of the silicon $(1 \mathrm{~mm})$. Photoexcitation thus introduces a very thin, partially conducting layer essentially at the surface of the silicon. Photoexcitation of the back interface decreases the intensity of the $\mathrm{THz}$ pulse that has already passed through the (unmodified) hole array by regulating the freecarrier absorption in the photoexcited layer. Accordingly, photoexcitation of the back interface does not alter the spectral profile of the transmitted pulse [Fig. 2(a)], and substantial intensities at $3.1 \mathrm{eV}$ are required for a significant drop in transmission at $\mathrm{THz}$ frequencies.

In contrast, photoexcitation of the front side [Fig. 2(b)] results not only in a substantially stronger photomodulation of the transmission; the spectral response is also modified (i.e., there is a spectral shift of the transmission peaks). For a relatively weak fluence of $0.1 \mathrm{~J} / \mathrm{m}^{2}$, excitation of the front interface reduces the transmission at resonance by $80 \%$, whereas for excitation of the back interface, transmission is only reduced by $20 \%$. This difference is highlighted in Fig. 2(c), where the dots indicate the absolute transmission at the $(1,0)$ peak after photoexciting the front and back interfaces. The trend in the data points for front excitation is clearly very different and can be fairly well reproduced by the model introduced in the next section. The transmission changes on photoexcitation also have a strong dependence on the spatial distribution of photoelectrons within the silicon surface layer at the gold interface, which is tunable through the wavelength of the excitation pulses: the transmission after front excitation by 3.1 and $1.5 \mathrm{eV}$ photons $\left(0.1 \mathrm{~J} / \mathrm{m}^{2}\right)$ is compared in Fig. 2(d). With $1.5 \mathrm{eV}$ photons we excite well below the direct band gap of silicon, resulting in a significantly larger penetration depth of the light $[\sim 1 \mu \mathrm{m}$ at $300 \mathrm{~K}$ (Ref. 25)], reducing the electron density (and conductivity) of the silicon at the hole array interface and reducing the photomodulation of transmission.

\section{MODELING}

Upon photoexcitation of the front of the sample, the silicon regions near the holes in the gold layer are made conducting. This will have two effects: (1) reducing the aperture scattering efficiency and the initial coupling to surface modes and (2) allowing surface modes at the silicon-gold interface to bridge the hole without scattering. Note that both of these mechanisms apply, irrespective of whether surface modes are described as spoof surface-plasmon polaritons ${ }^{12}$ or diffracted surface waves. ${ }^{13-16}$ As shown below, these two effects reduce the intensity in the diffracted modes on the silicon side of the grating.

We have carried out finite element method modeling with and without photoexcitation of our sample, approximating the photoexcited region as a $125 \mathrm{~nm}$ homogeneous layer below the holes. The intensity dependent changes in the dielectric function of this region are estimated from the incident excitation intensity using the Drude model according to Ref. 26. Figure 3(a) shows the time-averaged electric-field mag-
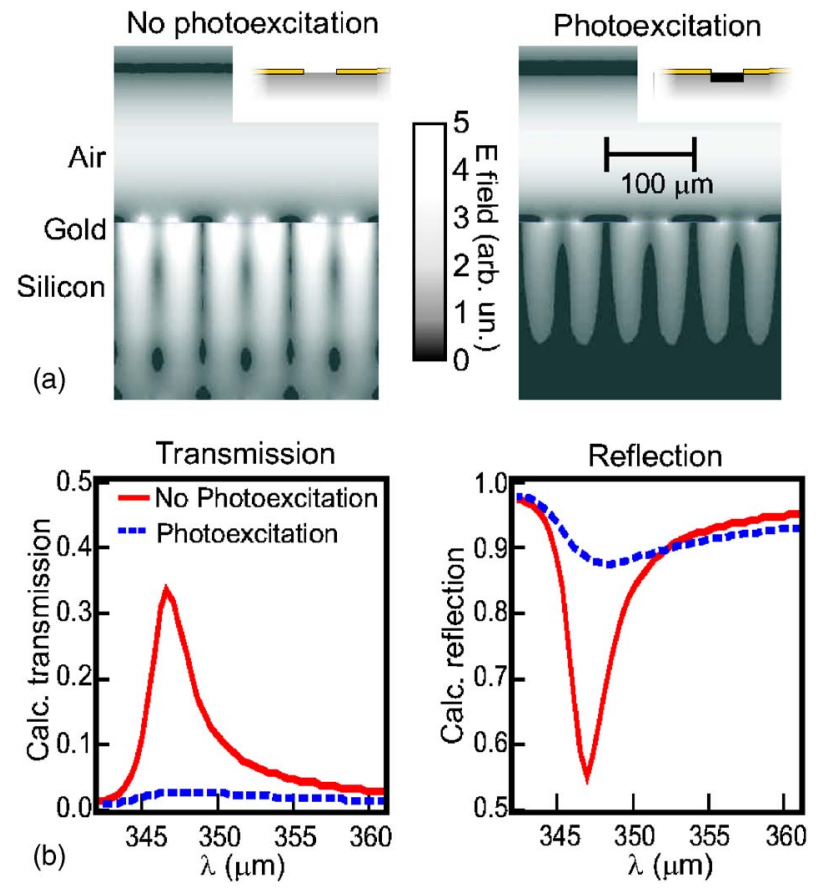

FIG. 3. (Color online) (a) Results of finite element method calculations showing the electric-field strength in the diffracted orders of the $(1,0)$ peak with and without photoexcitation by $0.1 \mathrm{~J} / \mathrm{m}^{2}$, $3.1 \mathrm{eV}$ pulses. The excitation region is treated as a homogeneous layer below the holes, $125 \mathrm{~nm}$ deep (dark region in the inset). (b) The change in diffracted intensity on photoexcitation is predominantly caused by an increase in reflection from the grating.

nitude for $\lambda=346 \mu \mathrm{m}$ [corresponding to the $(1,0)$ resonant transmission for the silicon-gold interface] with and without $0.1 \mathrm{~J} / \mathrm{m}^{2}, 3.1 \mathrm{eV}$ excitation. The modeling clearly shows that photoexcitation of the silicon reduces the electric-field strength in the light gray regions near the holes on the far side of the array (i.e., the diffracted orders on the silicon side of the grating).

In Fig. 3(b), we show the modeled reflection and transmission spectra, with and without photoexcitation of the front interface. The wavelength of resonant transmission agrees well with experiment, though the magnitude and width of transmission peaks are significantly larger and smaller, respectively, in the modeled data. We expect several effects to reduce the measured transmitted intensity and increase measured linewidths: these include beam focusing, limited frequency resolution, finite illumination area of sample, and sample inhomogeneities. Moreover, due to the finite computational power available, the modeled spectra in Fig. 3(b) are for reflection and transmission from the first interface of the sample only and, hence, do not take into account additional losses due to reflection from the second interface of the silicon wafer (around 30\%). Thus, while we expect the model to capture the essential characteristics of the measurement, a quantitative comparison of transmission magnitudes is difficult. Nevertheless, the modeled results clearly reproduce the redshift and broadening of transmission observed in the experiment upon photoexcitation [see vertical dotted lines in Fig. 2(b)]. Figure 3(b) shows that the large transmission changes on photoexcitation of the front sample 

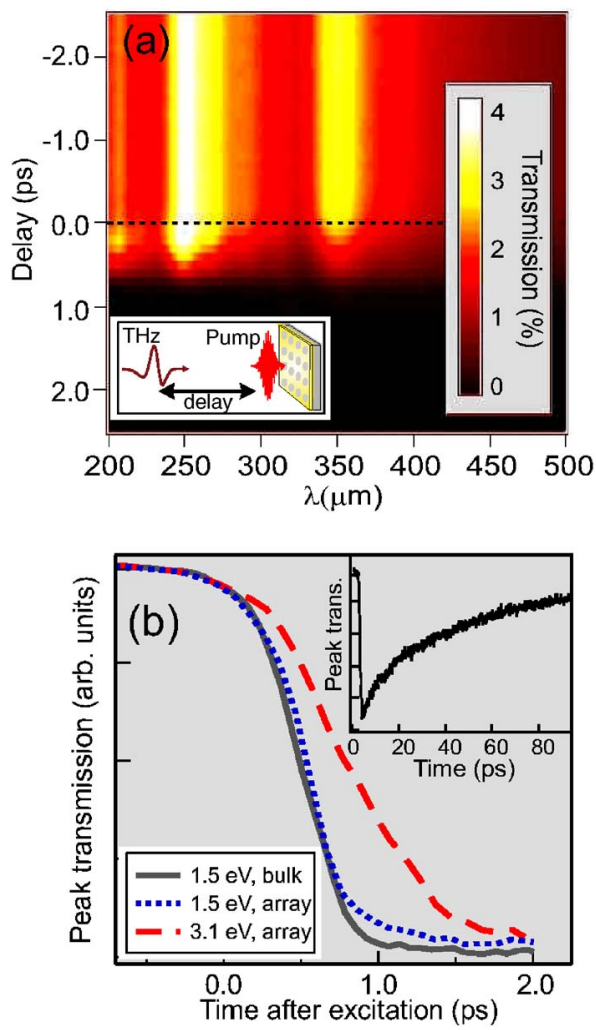

FIG. 4. (Color online) (a) Transmission spectrum measured as a function of time after excitation using $1 \mathrm{~J} / \mathrm{m}^{2}, 1.5 \mathrm{eV}$ photon pulses. (b) Comparison of switching dynamics: peak transmission (at $250 \mu \mathrm{m}$ ) as a function of time for bulk silicon (gray solid line) and hole array (blue/dark gray dotted line) after excitation by $1.5 \mathrm{eV}$ photons and hole array (red/light gray dashed line) after excitation by $3.1 \mathrm{eV}$ photons. Note that the data have been scaled and offset to allow direct comparison of the dynamics. Inset: recovery time for $0.1 \mathrm{~J} / \mathrm{m}^{2}$ at $20 \mathrm{~K}$.

interface are predominantly due to changes in reflection, and the model also reproduces the very marked fluence dependence for this effect [dotted line in Fig. 2(c)]. Furthermore, due to the focusing effect described above, we expect the reduction in transmission of a $\mathrm{THz}$ plane wave to be even stronger, more closely matching the lines of the model. Though the photoexcitation intensities required to reduce the $\mathrm{THz}$ transmission of the hole array are considerable $(\sim 0.1 \%$ of the damage threshold of silicon), they are significantly weaker than required to reduce $\mathrm{THz}$ transmission of a bare silicon surface (currently used as a photoactive switch for $\mathrm{THz}$ radiation ${ }^{27}$ ): the solid line in Fig. 2(c) represents the modeled transmission change for a photoexcited silicon surface, predominantly due to free-carrier absorption.

\section{SWITCHING DYNAMICS}

One of the main advantages that optical switching of transmission offers over thermal ${ }^{20}$ and magnetic ${ }^{21}$ switchings is fast switching times. In Fig. 4(a), we plot the transmission spectrum as a function of time after excitation with $1 \mathrm{~J} / \mathrm{m}^{2}$, $1.5 \mathrm{eV}$ photon pulses, measured and calculated according to Refs. 28 and 29: within one picosecond, resonant transmis- sion is reduced by almost 2 orders of magnitude. The blue dotted line in Fig. 4(b) represents a vertical cut of the data in Fig. 4(a) at $\lambda=346 \mu \mathrm{m}$ : the decay is exponential characterized by a time constant of $280 \mathrm{fs}$. Also plotted is the transmission dynamics of a pure silicon wafer under the same excitation conditions (solid gray line), scaled to match the array data. The similarity between the two traces suggests that the transmission dynamics of the hole array is determined by the bulk cooling of photoelectrons in silicon. We also observe that excitation with higher energy photons $\left(\sim 1 \mathrm{~J} / \mathrm{m}^{2}, 3.1 \mathrm{eV}\right.$ photon pulses) leads to slower dynamics (red dashed line), consistent with previous $\mathrm{THz}$ measurements of carrier cooling in bulk semiconductors. ${ }^{30}$ Therefore, by using photon energies exactly matched to the band gap of the material, it should be possible to reduce the transmission on even faster time scales, essentially limited by the temporal width of the excitation pulse. It should be noted, however, that switching times for any optical switch are restricted by the recovery time of transmission. Since silicon is an indirect band-gap semiconductor, photoconductivity decay (and transmission recovery) will involve trapping and eventual recombination at impurity centers. At low temperature and low carrier densities, this can be a fairly efficient process, ${ }^{31}$ and at $20 \mathrm{~K}$ and $0.1 \mathrm{~J} / \mathrm{m}^{2}$ excitation, we observe a subnanosecond recovery of transmission [see inset of Fig. 4(b)]. At room temperature, recovery times are significantly longer, extending to microseconds. Shorter recovery times at room temperature may be achieved by using a direct band-gap semiconductor. It should be noted that the switching times reported here are orders of magnitude faster than the (greater than milliseconds) switching times attainable for the thermal switching of semiconductor carrier densities ${ }^{20}$ and alignment of liquid crystals ${ }^{21}$ previously demonstrated for arrays.

\section{CONCLUSIONS}

To conclude, we have demonstrated ultrafast optical switching and some degree of tunability in the transmission of a subwavelength metal hole array in the $\mathrm{THz}$ frequency range. By fabricating the grating on a semiconductor substrate, visible light can be used to modify the electron density in the semiconductor and thereby control the $\mathrm{THz}$ transmission of the grating. We experimentally observe a factor of 2-5 improvement at resonance wavelengths in photomodulation efficiency over a bare silicon surface. The fast switching in transmission has implications for time-resolved and high bandwidth applications. Moreover, by photoexciting with photon energies close to the direct band gap of silicon [3.2 eV (Ref. 25)], transmission can be controlled with low light intensities.

\section{ACKNOWLEDGMENTS}

This work is part of the research program of the "Stichting voor Fundamenteel Onderzoek der Materie (FOM)," which is financially supported by the Nederlandse Organisatie voor Wetenschappelijk Onderzoek (NWO). The authors would like to thank F. J. Garcia Vidal, J. Bravo Abad, W. L. Barnes, and J. R. Sambles for helpful discussions. 
*FAX: $\quad+44 \quad 1392 \quad 264104 . \quad$ Electronic address: e.hendry@exeter.ac.uk

${ }^{1}$ T. W. Ebbesen, H. J. Lezec, H. F. Ghaemi, T. Thio, and P. A. Wolff, Nature (London) 391, 667 (1998).

${ }^{2}$ J. V. Coe, S. M. Williams, K. R. Rodriguez, S. Teeters-Kennedy, A. Sudnitsyn, and F. Hrovat, Anal. Chem. 78, 1384 (2006).

${ }^{3}$ W. L. Barnes, A. Dereux, and T. W. Ebbesen, Nature (London) 424, 824 (2003).

${ }^{4}$ J. A. H. van Nieuwstadt, M. Sandtke, R. H. Harmsen, F. B. Segerink, J. C. Prangsma, S. Enoch, and L. Kuipers, Phys. Rev. Lett. 97, 146102 (2006).

${ }^{5}$ X. G. Luo and T. Ishihara, Opt. Express 12, 3055 (2004).

${ }^{6}$ D. Qu, D. Grischkowsky, and W. Zhang, Opt. Lett. 29, 896 (2004).

${ }^{7}$ F. Miyamaru and M. Hangyo, Appl. Phys. Lett. 84, 2742 (2004).

${ }^{8}$ J. O'Hara, R. D. Averitt, and A. J. Taylor, Opt. Express 12, 6397 (2004).

${ }^{9}$ H. Cao and A. Nahata, Opt. Express 12, 3664 (2004).

${ }^{10}$ M. Beruete, M. Sorolla, I. Campillo, J. S. Dolado, L. MartínMoreno, J. Bravo-Abad, and F. J. García-Vidal, Opt. Lett. 29, 2500 (2004).

${ }^{11}$ H. Caglayan, I. Bulu, and E. Ozbay, Opt. Express 13, 1666 (2005).

${ }^{12}$ J. B. Pendry, L. Martin-Moreno, and F. J. Garcia-Vidal, Science 305, 847 (2004).

${ }^{13}$ M. M. J. Treacy, Appl. Phys. Lett. 75, 606 (1999).

${ }^{14}$ M. Sarrazin, J.-P. Vigneron, and J.-M. Vigoureux, Phys. Rev. B 67, 085415 (2003).

${ }^{15}$ H. Lezec and T. Thio, Opt. Express 12, 3629 (2004).

${ }^{16}$ F. J. García de Abajo, R. Gómez-Medina, and J. J. Sáenz, Phys.
Rev. E 72, 016608 (2005).

${ }^{17}$ J. Gómez Rivas, C. Schotsch, P. Haring Bolivar, and H. Kurz, Phys. Rev. B 68, 201306(R) (2003).

${ }^{18}$ C. Janke, J. Gómez Rivas, C. Schotsch, L. Beckmann, P. H. Bolivar, and H. Kurz, Phys. Rev. B 69, 205314 (2004).

${ }^{19}$ A. K. Azad, Y. Zhao, and W. Zhang, Appl. Phys. Lett. 86, 141102 (2005).

${ }^{20}$ J. Gómez Rivas, P. H. Bolivar, and H. Kurz, Opt. Lett. 29, 1680 (2004).

${ }^{21}$ C. L. Pan, C. F. Hsieh, R. P. Pan, M. Tanaka, F. Miyamaru, M. Tani, and M. Hangyo, Opt. Express 13, 3921 (2005).

${ }^{22}$ E. U. Donev, J. Y. Suh, F. Villegas, R. Lopez, R. F. Haglund, and L. C. Feldman, Phys. Rev. B 73, 201401(R) (2006).

${ }^{23}$ C. Janke, J. Gómez Rivas, P. H. Bolivar, and H. Kurz, Opt. Lett. 30, 2357 (2005).

${ }^{24}$ A. Nahata, A. S. Weling, and T. F. Heinz, Appl. Phys. Lett. 69, 2321 (1996).

${ }^{25}$ G. E. Jellison and F. A. Modine, Appl. Phys. Lett. 41, 180 (1982).

${ }^{26}$ E. Hendry, M. Koeberg, J. Pijpers, and M. Bonn (unpublished).

${ }^{27}$ F. A. Hegmann, J. B. Williams, B. Cole, M. S. Sherwin, J. W. Beeman, and E. E. Haller, Appl. Phys. Lett. 76, 262 (2000).

${ }^{28}$ E. Hendry, M. Koeberg, J. M. Schins, H. K. Nienhuys, V. Sunström, L. D. A. Siebbeles, and M. Bonn, Phys. Rev. B 71, 125201 (2005).

${ }^{29}$ J. T. Kindt and C. A. Schmuttenmaer, J. Chem. Phys. 110, 8589 (1999).

${ }^{30}$ N. Saeta, J. F. Federici, B. I. Greene, and D. R. Dykaar, Appl. Phys. Lett. 60, 1477 (1992).

${ }^{31}$ A. Hangleiter, Phys. Rev. B 35, 9149 (1987). 